\title{
Patient Preferences of Low-Dose Aspirin for Cardiovascular Disease and Colorectal Cancer Prevention in Italy: A Latent Class Analysis
}

\author{
Tommi Tervonen $^{1,2}$ (1) Pareen Vora ${ }^{3} \cdot$ Jaein Seo $^{4} \cdot$ Nicolas Krucien $^{1} \cdot$ Kevin Marsh ${ }^{1} \cdot$ Raffaele De Caterina $^{5,6}$. \\ Ulrike Wissinger $^{7} \cdot$ Montse Soriano Gabarró $^{3}$
}

Accepted: 28 February 2021 / Published online: 8 April 2021

(C) The Author(s) 2021

\begin{abstract}
Background Patients taking low-dose aspirin to prevent cardiovascular disease (CVD) may also benefit from a reduced risk of colorectal cancer (CRC).

Objective The aim was to examine the preferences of people eligible for preventive treatment with low-dose aspirin and the trade-offs they are willing to make between CVD prevention, CRC prevention, and treatment risks.

Methods A cross-sectional study using a discrete choice experiment (DCE) survey was conducted in Italy in 2019 to elicit preferences for three benefit attributes (prevention of ischemic stroke, myocardial infarction, and CRC) and four risk attributes (intracranial and gastrointestinal bleeding, peptic ulcer, and severe allergic reaction) associated with use of low-dose aspirin. Latent class logit models were used to evaluate variation in treatment preferences.

Results The DCE survey was completed by 1005 participants eligible for use of low-dose aspirin. A four-class model had the best fit for the primary CVD prevention group $(n=491)$, and a three-class model had the best fit for the secondary CVD prevention group $(n=514)$. For the primary CVD prevention group, where classes differed on age, education level, type 2 diabetes, exercise, and low-dose aspirin use, the most important attributes were intracranial bleeding (two classes), myocardial infarction (one class), and CRC (one class). For the secondary CVD prevention group, where classes differed on various comorbidities, self-reported health, exercise, and CVD medication use, the most important attributes were intracranial bleeding (two classes), myocardial infarction (one class), and gastrointestinal bleeding (one class).

Conclusion Patient preferences for the benefits and risks of low-dose aspirin differ significantly among people eligible for treatment as primary or secondary CVD prevention.
\end{abstract}

\section{Key Points for Decision Makers}

Patient preferences for the benefits and risks of lowdose aspirin differ among people eligible for treatment

Tommi Tervonen

Tommi.Tervonen@evidera.com as primary or secondary cardiovascular disease (CVD) prevention.

Patient-Centered Research, Evidera, London, UK

2 Department of Epidemiology, University Medical Center Groningen, University of Groningen, Groningen, The Netherlands

3 Epidemiology, Bayer AG, Berlin, Germany

4 Patient-Centered Research, Evidera, Bethesda, MD, USA

5 Cardiology Division, University of Pisa, Pisa University Hospital, Pisa, Italy

6 Fondazione VillaSerena per la Ricerca, Città Sant'Angelo, Pescara, Italy

7 Medical Affairs, Bayer AG, Berlin, Germany

The potential colorectal cancer prevention benefit may be especially important to some younger patients eligible for low-dose aspirin for primary prevention of CVD. 


\section{Introduction}

Low-dose aspirin (75-325 mg/day) is one of the most widely used treatments for prevention of cardiovascular (CV) events. Treatment guidelines and recommendations advocate the use of low-dose aspirin for secondary prevention of cardiovascular disease (CVD) and for primary prevention in people at high risk of CV events $[1,2]$. An added benefit of low-dose aspirin is protection against colorectal cancer (CRC) [3-5]. A systematic review of the literature and a meta-analysis found that aspirin at any dose may be effective at preventing CRC [5, 6]. A benefit-risk analysis based on the systematic review estimated that for average-risk individuals taking low-dose aspirin prophylactically for 10 years, approximately one third of the overall benefit would be due to the reduction in CRC incidence [6]. Currently, the US Preventive Services Task Force recommends low-dose aspirin for the primary prevention of both CVD and CRC in adults aged 50-59 years who have a 10 -year CVD risk $\geq 20 \%$ and in selected adults aged 60-69 years [7].

Low-dose aspirin, however, can be associated with adverse effects, such as gastrointestinal bleeding and, less frequently, intracranial bleeding, gastrointestinal ulcers, and anaphylactic reactions [8-10]. Nonetheless, benefit-risk analyses suggest that low-dose aspirin provides more benefits than risks, especially in individuals who are at high risk of CVD and low risk of gastrointestinal bleeding $[1,11,12]$. The greatest potential benefit in life years and quality-adjusted life years of using low-dose aspirin for primary prevention of CVD and CRC is gained when preventive treatment is initiated earlier (40-69 years of age) by patients and maintained for the remainder of their lives [13].

To be able to recommend appropriate preventative treatment to people at risk of $\mathrm{CV}$ events, decision makers need to understand how individuals value aspects such as effectiveness, safety, and convenience $[14,15]$. Benefit-risk analyses can provide an objective assessment of the relative benefits and risks of treatments, but they do not provide information on how important different treatment attributes are to patients. Quantitative preference elicitation methods are needed to provide a deeper understanding of patients' preferences and their willingness to make trade-offs between benefits and risks [16]. Previous patient preference studies on primary or secondary prevention of CV events that have used DCE surveys have generally shown that patients highly value reducing the risk of ischaemic stroke, although results have been variable and prevention of CRC has not been included as a potential benefit [17-21].
In the present study, we aimed to understand how people taking or eligible for low-dose aspirin for primary or secondary prevention of CVD value the key CVD prevention benefits and treatment risks, as compared to the CRC prevention benefit; how these preferences vary between individuals; and the contribution of personal characteristics to the variation.

\section{Methods}

\subsection{Overall Study Design}

This was a cross-sectional study conducted in Italy using a discrete choice experiment (DCE) as a stated-preference method. DCEs are based on the idea that the value people place on different options depends on the attributes characterizing them [22, 23], for example, for different treatment options, their levels of efficacy and adverse effects. We applied a mixed-methods approach that combined qualitative patient and physician interviews with a quantitative patient preference elicitation using a DCE, which was conducted following good research practices [24, 25]. Benefit and risk attributes relevant to low-dose aspirin were first identified through a targeted literature review (Online Resource 1, see the electronic supplementary material). We reviewed current practice guidelines of the United States Preventative Services Task Force, European Society of Cardiology, World Health Organization, American College of Chest Physicians, and American Heart Association, as well as published literature, which was identified by searching major medical databases for both qualitative and quantitative preference studies on the use of aspirin, antiplatelet drugs, and nonsteroidal anti-inflammatory drugs in primary or secondary prevention of CVD or CRC. The published literature used to identify potential attributes and levels is summarized in Online Resource 1.

The relevance of the identified attributes was explored in qualitative telephone interviews with ten Italian-speaking individuals taking or eligible for low-dose aspirin and three Italian-speaking, medically trained physicians, each with $\geq 5$ years' experience of prescribing low-dose aspirin for primary and secondary CVD prevention. The aim was to understand why certain benefits and risks of low-dose aspirin are considered important and the willingness to make trade-offs among them. The interviews were conducted using a semistructured interview guide, and with an emphasis on CVD and CRC prevention. Patients were asked for their perspectives on the benefits and risks of using low-dose aspirin, and physicians were asked about issues and concerns with prescribing low-dose aspirin. In these qualitative interviews, the selected attributes were endorsed to be relevant and no other relevant attributes were found to be missing. 
The final attributes and levels were implemented in a DCE survey that was tested with ten Italian-speaking participants taking or eligible for low-dose aspirin for primary or secondary CVD prevention. A semi-structured interview guide was used to determine whether the attributes and levels were understandable to participants; to assess participants' ability to understand the DCE and distinguish between levels; and to confirm that the DCE survey was user friendly. After the first five interviews, changes were made to the wording and organization of the DCE survey. The DCE survey was then tested on participants in a second set of five interviews. Further minor changes were made after the second set of interviews. The final web-based DCE survey was fielded in August to December 2019.

\subsection{Participants}

The study enrolled two groups of participants: adults aged $50-75$ years eligible to receive low-dose aspirin $(100 \mathrm{mg} /$ day) for primary prevention of CVD and adults aged $\geq 18$ years eligible to receive low-dose aspirin for secondary prevention of CVD. Eligibility for the primary CVD prevention group was based on a 10-year CVD risk of $\geq 20 \%$ [11]. CVD risk was estimated from self-reported clinical characteristics using the Progetto Cuore risk chart, which assesses the probability of developing a first major CV event based on sex, age, diabetes status, smoking status, systolic blood pressure, and serum total cholesterol level [26]. Eligibility for the secondary CVD prevention group was based on self-reported history of myocardial infarction, ischaemic stroke, transient ischaemic stroke, or angina.

Participants were recruited from two online general population panels with a combined membership of 250,000 in Italy, as well as through physician referrals. Potential participants were contacted by email and screened through a webbased questionnaire. All participants had to be able to read and understand Italian and not be participating in an interventional study. Individuals were ineligible if they belonged to a group for which aspirin use is not recommended (i.e. if they had glucose-6-phosphate dehydrogenase deficiency or another condition for which low-dose aspirin is contraindicated, were being treated with methotrexate, or were pregnant); were employed by a pharmaceutical company; had a direct role in treating patients with CVD; or had a cognitive impairment, hearing difficulty, visual impairment, or acute psychopathology that, in the investigator's or interviewer's judgement, could interfere with the ability to provide online consent and complete an interview or survey. Eligible participants who provided online consent were presented with the objective of the study, a description of the survey structure, and descriptions of the attributes and levels. They then completed the DCE, questions to assess their health literacy and numeracy using validated scales [27, 28], and questions on demographics and medical history.

\subsection{DCE}

Thirty-six DCE questions were generated using a D-efficient design, which were divided across three blocks with 12 questions each and randomly allocated to participants. Each DCE question presented participants with two hypothetical treatments and a no-treatment option. The three options were defined according to seven attributes: three benefit attributes (decreased risks of ischaemic stroke, myocardial infarction, and $\mathrm{CRC}$ ) and four risk attributes reflecting potential adverse effects of low-dose aspirin treatment (increased risks of gastrointestinal bleeding, peptic ulcer, intracranial bleeding, and severe allergic reaction) (Table 1), which were introduced to participants before the DCE (Online Resource 2, see the electronic supplementary material). The order of the DCE questions and the order in which individual benefit and risk attributes were presented was randomized among participants, although the benefit attributes were always presented before the risk attributes. An example question is shown in Fig. 1.

In addition to the 12 DCE questions used for capturing participant preferences, two non-experimental questions were included to assess participant attention to the choice questions: a repeated question and a dominance question. The first question was repeated as the seventh question to assess whether participants provided consistent answers. The last question was a dominance question where the no-treatment option had the lowest risk levels for ischemic stroke, myocardial infarction, and CRC and no risk for the adverse effect attributes, and where the risk levels for treatment options A and B were higher for at least one attribute. This question was used to assess whether participants understood and paid attention to the discrete choice task by choosing the no-treatment option.

\subsection{Statistical Analyses}

Statistical analyses were performed using $\mathrm{R}$ version 3.6.1 [29], with the packages 'mlogit' [30] and 'gmnl' [31] for analyses of discrete choice data. All participants who fully completed the survey were included in the analyses. Sociodemographic and clinical data were summarized as descriptive statistics.

\subsubsection{Latent Class Logit Analysis}

Latent class logit (LCL) models assume that preferences differ across participants and that participants can be grouped probabilistically according to distinct preference classes, each corresponding to a unique pattern of treatment 
Table 1. Attributes and levels in the DCE

\begin{tabular}{|c|c|c|}
\hline \multirow[t]{2}{*}{ Attribute $^{a}$} & \multicolumn{2}{|l|}{ Levels } \\
\hline & Primary CVD prevention & Secondary CVD prevention \\
\hline \multirow[t]{3}{*}{ Ischaemic stroke (10-year risk) } & 5 out of 100 & 15 out of 100 \\
\hline & 10 out of 100 & 20 out of 100 \\
\hline & 15 out of $100^{c}$ & 30 out of $100^{c}$ \\
\hline \multirow[t]{3}{*}{ Myocardial infarction (10-year risk) } & 10 out of 100 & 40 out of 100 \\
\hline & 15 out of 100 & 50 out of 100 \\
\hline & 20 out of $100^{c}$ & 60 out of $100^{c}$ \\
\hline \multirow[t]{3}{*}{ Colorectal cancer (10-year risk) } & 1 out of 100 & \\
\hline & 2 out of 100 & \\
\hline & 3 out of $100^{c}$ & \\
\hline \multirow[t]{2}{*}{ Gastrointestinal bleeding } & No risk ${ }^{b}$ & \\
\hline & Increased risk & \\
\hline \multirow[t]{2}{*}{ Peptic ulcer } & No risk ${ }^{b}$ & \\
\hline & Increased risk & \\
\hline \multirow[t]{2}{*}{ Intracranial bleeding } & No risk ${ }^{b}$ & \\
\hline & Increased risk & \\
\hline \multirow[t]{2}{*}{ Severe allergic reaction } & No risk ${ }^{b}$ & \\
\hline & Increased risk & \\
\hline
\end{tabular}

$C V D$ cardiovascular disease, $D C E$ discrete choice experiment

${ }^{a}$ The following plain language terms were used in the actual survey: stroke (= ischaemic stroke), heart attack (= myocardial infarction), stomach bleeding (= gastrointestinal bleeding), stomach ulcer (= peptic ulcer), and bleeding in the brain (= intracranial bleeding)

${ }^{\mathrm{b}}$ This level was selected for the no-treatment option

${ }^{\mathrm{c}}$ Indicates a reference level

preferences. In this study, LCL models were fitted separately for the primary and secondary CVD prevention groups. The optimal number of latent classes was determined by fitting LCL models with an increasing number of classes (from 2 to 6 ) and selecting the one where the Bayesian information criterion was minimized (Online Resource 3, see the electronic supplementary material). Log-likelihood, Akaike information criterion (AIC), AIC3, corrected AIC, and consistent AIC were also calculated.

For each class, maximum likelihood estimates reflecting preferences for marginal changes in the attributes were obtained, together with their standard error, and statistical significance of the estimated preferences was determined by two-sided $z$-test at the $5 \%$ level. The utility function specified in each model was additive, meaning that the preferences for different attributes were assumed to be independent. The assumption of linearity in preferences for the three benefit attributes (decreased risks of ischaemic stroke, myocardial infarction, and CRC) was tested in linear regression models using multinomial logit model preference estimates and was found to be acceptable, with $R^{2}$ in the range $77-100 \%$ for the primary CVD prevention group and $97-100 \%$ for the secondary CVD prevention group. The benefit attributes were then entered as continuous variables in the model, which measured the preference for a $1 \%$ decrease in risk. The four risk attributes (risks of gastrointestinal bleeding, peptic ulcer, intracranial bleeding, and severe allergic reaction) were entered as binary variables in the model, which measured the preference for a change from "increased risk" to "no risk". The model also included two constant terms to capture preferences for the no-treatment option and for option A. The latter constant was used to control for potential left-right bias, although as the order of treatment options A and B was not randomized between participants, it may also have captured statistical artefacts of the design.

Model estimates were used to compute relative importance (RI) scores for each attribute in each class. The RI scores, which indicate the value of change across the full range of levels for each individual attribute, were illustrated graphically as radar charts. Confidence intervals for the RI scores were calculated by the Krinsky-Robb procedure [32, 33] using 10,000 draws for the simulation, and Wald tests were used to compare RI profiles across latent classes.

The LCL model estimates were then used to compute the probability of each participant belonging to the different classes and participants were allocated to the class to which 
they had the highest probability of belonging. Chi-square test of sample proportions was used to detect significant differences in the personal characteristic compositions of the latent classes. Only characteristics achieving a prevalence of at least $10 \%$ observations in each modality were considered. A personal characteristic was identified as a potential predictor of preference heterogeneity when the chi-square test was significant at the $5 \%$ level.

\subsubsection{Data Validity Assessment}

Lexicographic preferences were assessed by determining whether participants always chose the treatment option that was better on one of the attributes. Serial non-participation was assessed by determining whether participants always chose treatment option A, treatment option B, or the no-treatment option. Understanding and attention to the DCE task was assessed by determining whether participants selected the dominant no-treatment option in the dominance question. Choice stability was assessed by determining if participants provided the same answer each time to the repeated question.

\section{Results}

\subsection{Participants}

Of 5134 individuals invited to participate, 2332 completed the screening questionnaire (Fig. 2). The DCE survey was completed by 1005 participants (20\% of those invited to participate). Of these, 491 were eligible for or taking lowdose aspirin for primary CVD prevention and 514 were eligible for or taking low-dose aspirin for secondary CVD prevention. In the primary CVD prevention group, the most frequent reasons for study exclusion were having a CV risk $<20 \%(n=833)$, unknown systolic blood pressure $(n=107)$, and unknown cholesterol level $(n=130)$. In the secondary CVD prevention group, the most common reason for study exclusion was having disqualifying comorbidities $(n=28)$.

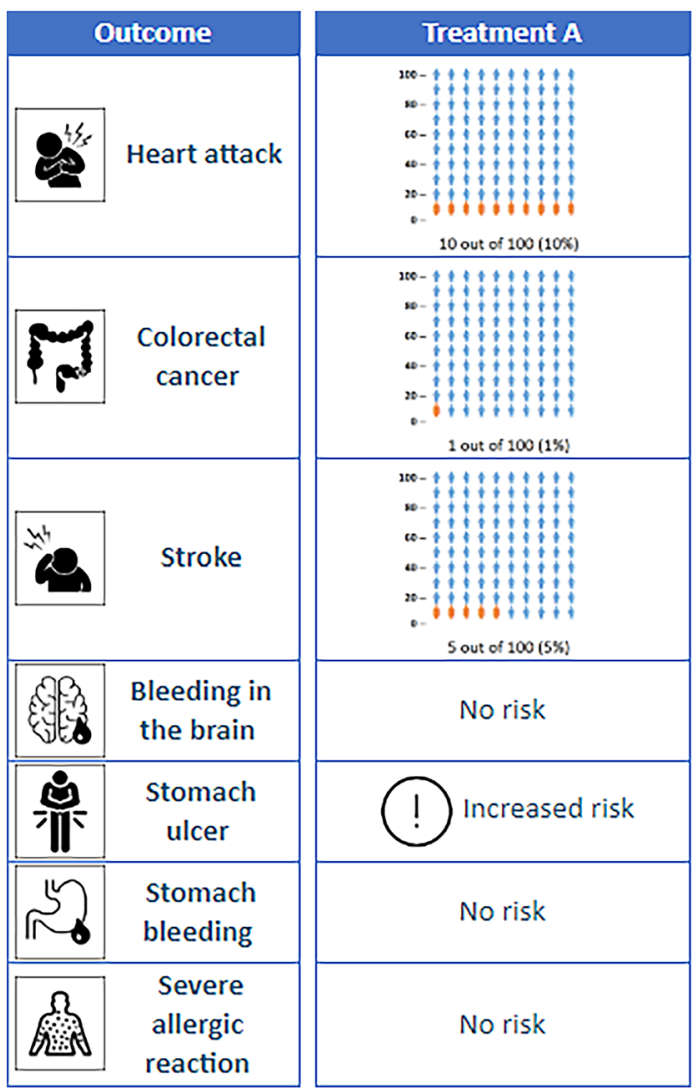

Fig. 1. Example choice question from the survey for primary prevention group. The following plain language terms were used: heart attack (= myocardial infarction), stroke (= ischaemic stroke), bleed-

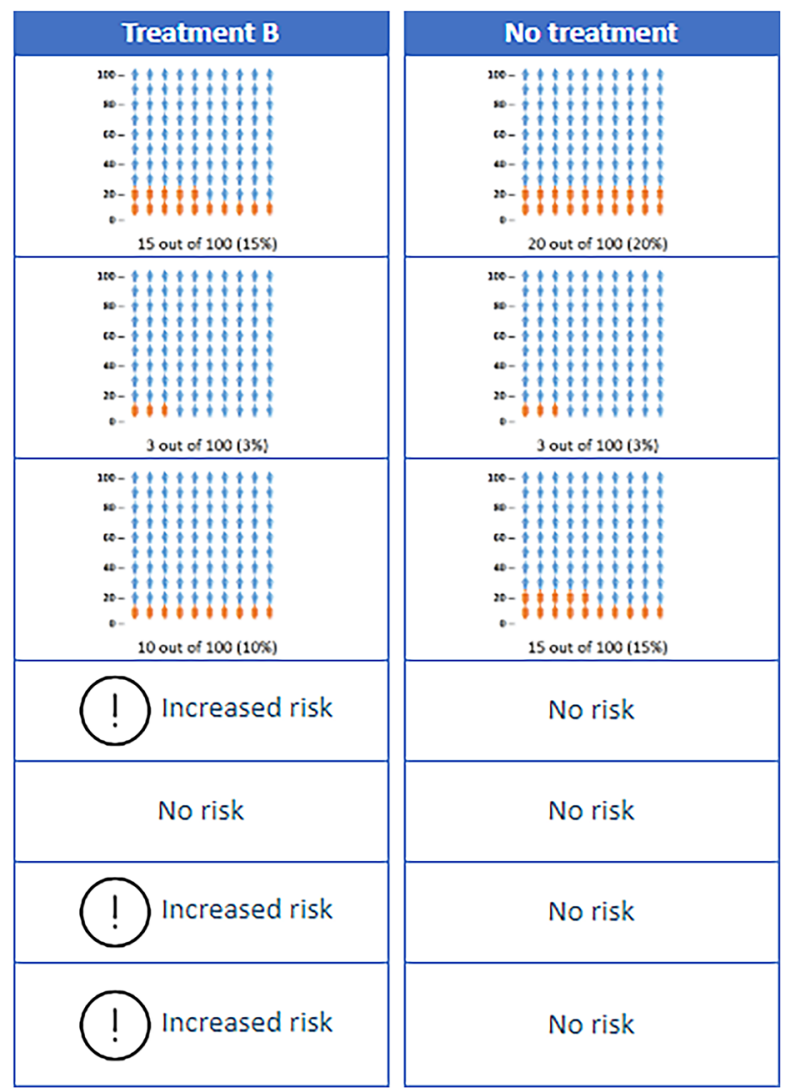

ing in the brain (= intracranial bleeding), stomach ulcer (= peptic ulcer), and stomach bleeding (= gastrointestinal bleeding) 
Fifty-one per cent of participants in the primary CVD prevention group and $48 \%$ in the secondary CVD prevention group were female (Table 2). Mean age was 62.5 years for the primary CVD prevention group and 62.2 years for the secondary CVD prevention group. Most participants (72\% in the primary CVD prevention group and $75 \%$ in the secondary CVD prevention group) were married. Only $24 \%$ had at least college education. Mean body mass index was $26.8 \mathrm{~kg} /$ $\mathrm{m}^{2}$ for the primary CVD prevention group and $26.4 \mathrm{~kg} / \mathrm{m}^{2}$ for the secondary CVD prevention group. Fifty-two per cent of participants in the primary CVD prevention group and $58 \%$ in the secondary CVD prevention group were current or former smokers.

Frequent comorbidities included type 2 diabetes, reported by $41 \%$ of participants in the primary CVD prevention group and $20 \%$ of participants in the secondary CVD prevention group. In the secondary CVD prevention group, myocardial infarction was reported by $36 \%$ of participants, stable angina by $31 \%$, heart arrhythmias by $29 \%$, transient ischemic attack by $27 \%$, atherosclerosis by $16 \%$, unstable angina by $14 \%$, and ischaemic stroke by $11 \%$. Low-dose aspirin was being taken by $47 \%$ of participants in the primary CVD prevention group and $50 \%$ of participants in the secondary CVD prevention group.

Based on their answers to the health literacy and numeracy questions, $91 \%$ of participants had adequate numeracy but only $35 \%$ had adequate health literacy. Ninety-four per cent of participants answered the dominance question correctly, and $66 \%$ gave the same answers to the repeated question. Fourteen per cent of participants showed lexicographic preferences (always choosing the treatment option that was better on one of the attributes), most frequently for intracranial bleeding (9\%). One percent of participants always chose treatment $\mathrm{A}$ or always chose treatment $\mathrm{B}$, and $14 \%$ always chose the no-treatment option (Online Resource 4, see the electronic supplementary material).

\subsection{Participant preferences for treatment attributes}

\subsubsection{Primary CVD Prevention Group}

A four-class model (Bayesian information criterion $=8945$ ) best explained the variability in participants' choices for the primary CVD prevention group (Online Resource 5, see the electronic supplementary material). Wald tests indicated that the RI values differed significantly between all classes ( $p=$ 0.043 or less; Online Resource 6). Participants in class 1 (37\% of participants) had lower education than participants in other classes (21\% with higher education vs. $33-37 \%$ ) (Online Resource 7). The most important treatment attribute for participants in class 1 was intracranial bleeding $(\mathrm{RI}=$ $28 \%$ ) (Fig. 3). For class 2 (26\% of participants), a greater proportion of participants were taking low-dose aspirin for a heart condition than participants in other classes (56\% vs. 40-51\%). The most important attributes for class 2 were myocardial infarction $(\mathrm{RI}=35 \%)$ and ischaemic stroke (RI $=29 \%$ ). Participants in class 3 (19\% of participants) exercised less than participants in other classes (38\% no exercise/ week vs. $27-33 \%, 24 \% \geq 3 \mathrm{~h}$ exercise/week vs. $32-36 \%$ ). The most important attributes for class 3 were intracranial bleeding $(\mathrm{RI}=32 \%)$ and gastrointestinal bleeding $(\mathrm{RI}=$ $23 \%$ ). Lastly, the most important attributes for participants in class 4 ( $18 \%$ of participants), who were younger $(27 \% \leq 55$ years vs. $13-16 \%, 31 \% \geq 65$ years vs. $44-48 \%$ ) and had less type 2 diabetes ( $30 \%$ vs. 38-48\%) than participants in other classes, were CRC (RI $=25 \%)$ and intracranial bleeding $(\mathrm{RI}=20 \%)$.

\subsubsection{Secondary CVD Prevention Group}

For the secondary CVD prevention group, a three-class model (Bayesian information criterion $=7843$ ) best explained the variability in participants' choices (Online Resource 8 , see the electronic supplementary material). Wald tests indicated that the RI values differed significantly between all classes ( $p<0.001$; Online Resource 6). Participants in class 1 (37\% of participants) were younger than those in other classes $(31 \% \leq 55$ years vs. $12-16 \%, 15 \%$ $\geq 65$ years vs. $57-64 \%$ ), and more participants in class 1 had inadequate health literacy (87\% vs. 60-65\%) and a lower level of education (37\% primary school vs. $28-33 \%$, $12 \%$ higher education vs. 19-25\%) (Online Resource 9). There were more current and former smokers in class $1(9 \%$ never smoked vs. 53-67\%), and fewer participants in class $1 \mathrm{did} \geq 3 \mathrm{~h}$ exercise/week ( $2 \%$ vs. $20-32 \%$ ) compared to other classes. Class 1 also had higher rates of comorbidities (42\% congestive heart failure vs. $2-10 \%, 34 \%$ deep vein thrombosis vs. $0-7 \%, 66 \%$ heart arrhythmias vs. 3-15\%, $40 \%$ heart valve problems vs. $2-8 \%, 94 \%$ hypertension vs. 60-75\%, 89\% hypercholesterolemia vs. 51-58\%, 40\% transient ischemic attack vs. 16-23\%, 24\% unstable angina vs. 9-11\%, 31\% kidney failure vs. $1 \%$, and $26 \%$ type 2 diabetes vs. $15-18 \%$ ), and hospitalization (83\% vs. 13-26\%). Fewer participants in class 1 had been taking medications for a heart condition for $>5$ years $(12 \%$ vs. $76-77 \%)$, had previously taken anticoagulants or antiplatelets other than aspirin for a heart condition ( $6 \%$ vs. 19-29\%), and had a history of myocardial infarction ( $22 \%$ vs. $43-46 \%$ ) or ischaemic stroke (6\% vs. 13-15\%). The most important attributes for participants in class 1 were gastrointestinal bleeding $(\mathrm{RI}=31 \%)$ and intracranial bleeding $(\mathrm{RI}=31 \%$ ) (Fig. 4). Participants in class 2 (35\% of participants) had better self-reported health than those in other classes (57\% fair/poor health vs. $68 \%$ ) and more of them reported doing $\geq 3 \mathrm{~h}$ of exercise per week (32\% vs. 2-20\%). The most important treatment attribute for participants in class 2 was myocardial infarction $(\mathrm{RI}=28 \%)$. 
Table 2. Demographic and clinical characteristics of participants

\begin{tabular}{|c|c|c|}
\hline Characteristic & $\begin{array}{l}\text { Primary CVD prevention } \\
(n=491)\end{array}$ & $\begin{array}{l}\text { Secondary CVD prevention } \\
(n=514)\end{array}$ \\
\hline \multicolumn{3}{|l|}{ Sex, $n(\%)$} \\
\hline Female & $248(51)$ & $247(48)$ \\
\hline Age (years), mean (standard deviation) & $62.5(6.3)$ & $62.2(6.6)$ \\
\hline \multicolumn{3}{|l|}{ Marital status, $n(\%)$} \\
\hline Married & $355(72)$ & $387(75)$ \\
\hline Widowed & $36(7)$ & $45(9)$ \\
\hline Divorced & $22(4)$ & $30(6)$ \\
\hline Separated & $30(6)$ & $25(5)$ \\
\hline Single & $48(10)$ & $27(5)$ \\
\hline \multicolumn{3}{|l|}{ Education, $n(\%)$} \\
\hline Primary school & $78(16)$ & $168(33)$ \\
\hline High school & $270(55)$ & $250(49)$ \\
\hline College or higher & $143(29)$ & $96(19)$ \\
\hline \multicolumn{3}{|l|}{ Body mass index $\left(\mathrm{kg} / \mathrm{m}^{2}\right)$} \\
\hline Mean (standard deviation) & $26.8(5.7)$ & $26.4(4.3)$ \\
\hline Missing, $n(\%)$ & $19(4)$ & $76(15)$ \\
\hline \multicolumn{3}{|l|}{ Smoking status, $n(\%)$} \\
\hline Current smoker & $222(45)$ & $144(28)$ \\
\hline Former smoker & $32(7)$ & $156(30)$ \\
\hline Never smoked & $237(48)$ & $214(42)$ \\
\hline \multicolumn{3}{|l|}{ Current medical conditions, $n(\%)$} \\
\hline Atherosclerosis & $19(4)$ & $84(16)$ \\
\hline Cancer other than colorectal cancer & $6(1)$ & $4(1)$ \\
\hline Colorectal cancer & $3(1)$ & 0 \\
\hline Congestive heart failure & $8(2)$ & $98(19)$ \\
\hline Diabetes, type 1 & $23(5)$ & $4(1)$ \\
\hline Diabetes, type 2 & $200(41)$ & $103(20)$ \\
\hline Heart arrhythmias & $17(3)$ & $151(29)$ \\
\hline Heart valve problems & $9(2)$ & $89(17)$ \\
\hline High cholesterol & $465(95)$ & $344(67)$ \\
\hline Hypertension & $464(95)$ & $394(77)$ \\
\hline Myocardial infarction & 0 & $185(36)$ \\
\hline Peripheral arterial disease & $5(1)$ & $23(4)$ \\
\hline Stable angina & 0 & $159(31)$ \\
\hline Ischaemic stroke & 0 & $58(11)$ \\
\hline Transient ischemic attack & 0 & $137(27)$ \\
\hline Unstable angina & 0 & $71(14)$ \\
\hline \multicolumn{3}{|l|}{ Current heart medications, $n(\%)^{\mathrm{a}}$} \\
\hline Cholesterol-lowering drugs & $420(86)$ & $367(71)$ \\
\hline Antihypertensive drugs & $444(90)$ & $397(77)$ \\
\hline Low-dose aspirin & $230(47)$ & $258(50)$ \\
\hline $\begin{array}{l}\text { Other antiplatelets or anticoagu- } \\
\text { lants Other than aspirin }\end{array}$ & $13(3)$ & $193(38)$ \\
\hline Other $^{b}$ & $14(3)$ & $11(2)$ \\
\hline None & 0 & 0 \\
\hline
\end{tabular}

CVD cardiovascular disease

anformation on current heart medications was collected with the question "Which of the following medications are you currently taking for your heart disease?"

b Based on participant responses for a free-text "Other" response option 


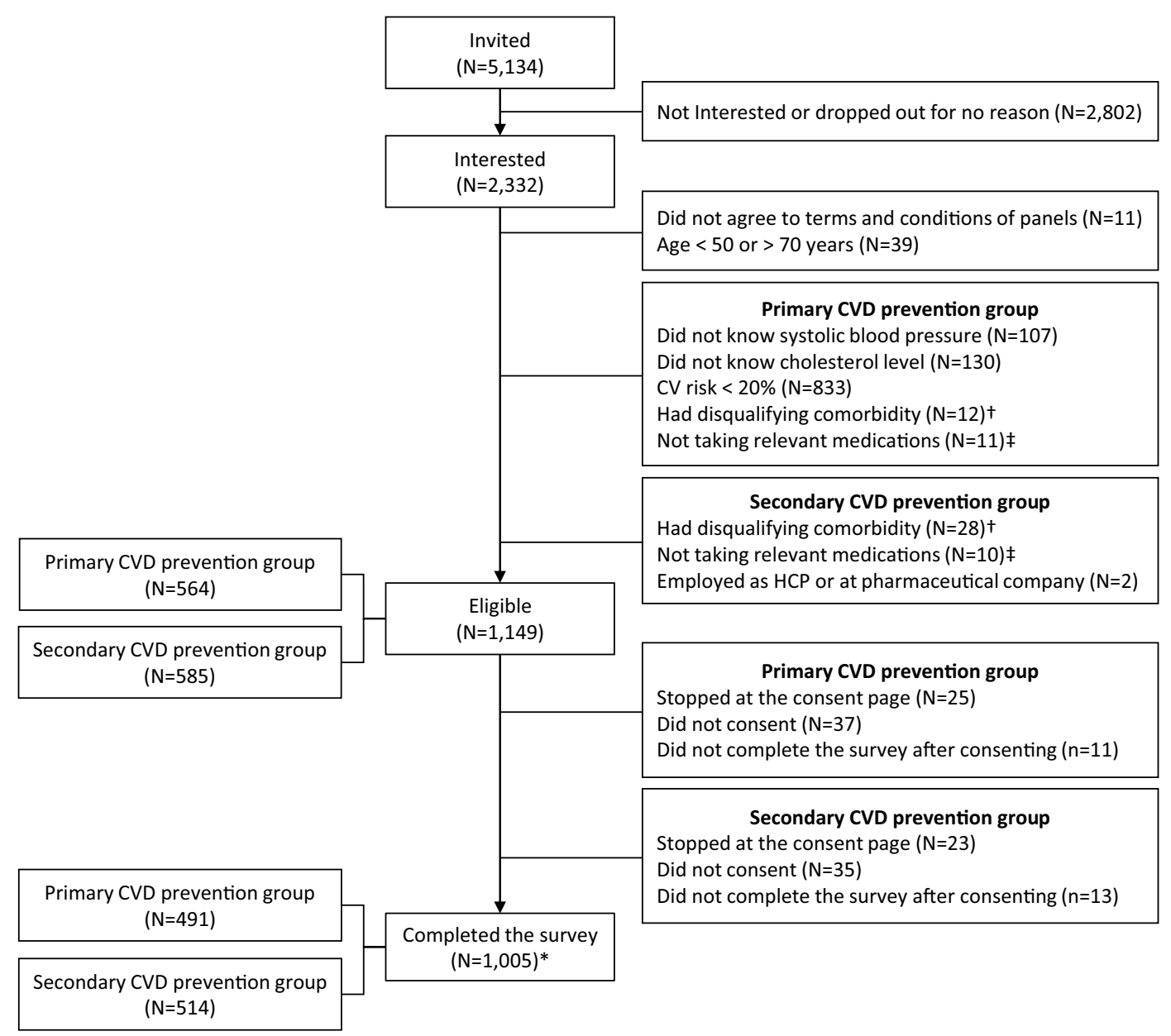

Fig. 2. Participant disposition. $C V$ cardiovascular, $C V D$ cardiovascular disease, $H C P$ health care professional. *A total of $807(80 \%)$ recruited from panels and 198 (20\%) recruited from HCP referrals. ${ }^{\dagger}$ Disqualifying comorbidities included glucose-6-phosphate dehydro-

And for class 3 ( $28 \%$ of participants), more participants had previously used anticoagulants or antiplatelets other than aspirin compared to other classes ( $29 \%$ vs. $6-19 \%$ ), although current use of anticoagulants and antiplatelets did not differ between the classes ( $p=0.459$ for low-dose aspirin, $p$ $=0.977$ for other drugs). The most important attribute for class 3 was intracranial bleeding $(\mathrm{RI}=28 \%)$. CRC prevention was the least important attribute for all secondary CVD prevention classes $(\mathrm{RI}=1-8 \%)$.

\section{Discussion}

Treatment guidelines recommend low-dose aspirin for secondary prevention of CVD and for primary prevention of CVD in people at high risk of $\mathrm{CV}$ events and acceptable risk of bleeding $[1,2]$, as well as for primary prevention of CRC [7]. Formal benefit-risk analyses suggest that the benefits genase deficiency, cognitive impairment, visual impairment, hearing difficulty and acute psychopathology. ${ }^{\star}$ Relevant medications required to take included cholesterol-lowering drugs, anti-hypertensive drugs, low-dose aspirin and antiplatelets or anticoagulants other than aspirin

of low-dose aspirin outweigh the risks $[12,13]$. However, being able to recommend appropriate preventive treatments to people at risk of $\mathrm{CV}$ events partly depends on understanding how individuals value treatment attributes. Patients and physicians often value the benefits and risks of prophylactic treatments differently [34-36]. For CVD treatments, patients are less willing than physicians to accept some of the common risks [20].

Patient preference studies have repeatedly found that reducing the risk of CVD (e.g. stroke and myocardial infarction) and avoiding adverse effects of treatment such as bleeding are important treatment considerations for people at risk of CVD [17-19, 21, 37, 38]. The frequency and costs of treatment are also important to patients [39]. The current study, conducted in Italy, where low-dose aspirin $(100 \mathrm{mg} /$ day $)$ is recommended for primary and secondary CVD prevention, found that preferences towards the treatment benefits and potential adverse effects of low-dose 
Fig. 3. Relative importance by preference class for the primary cardiovascular disease prevention group.

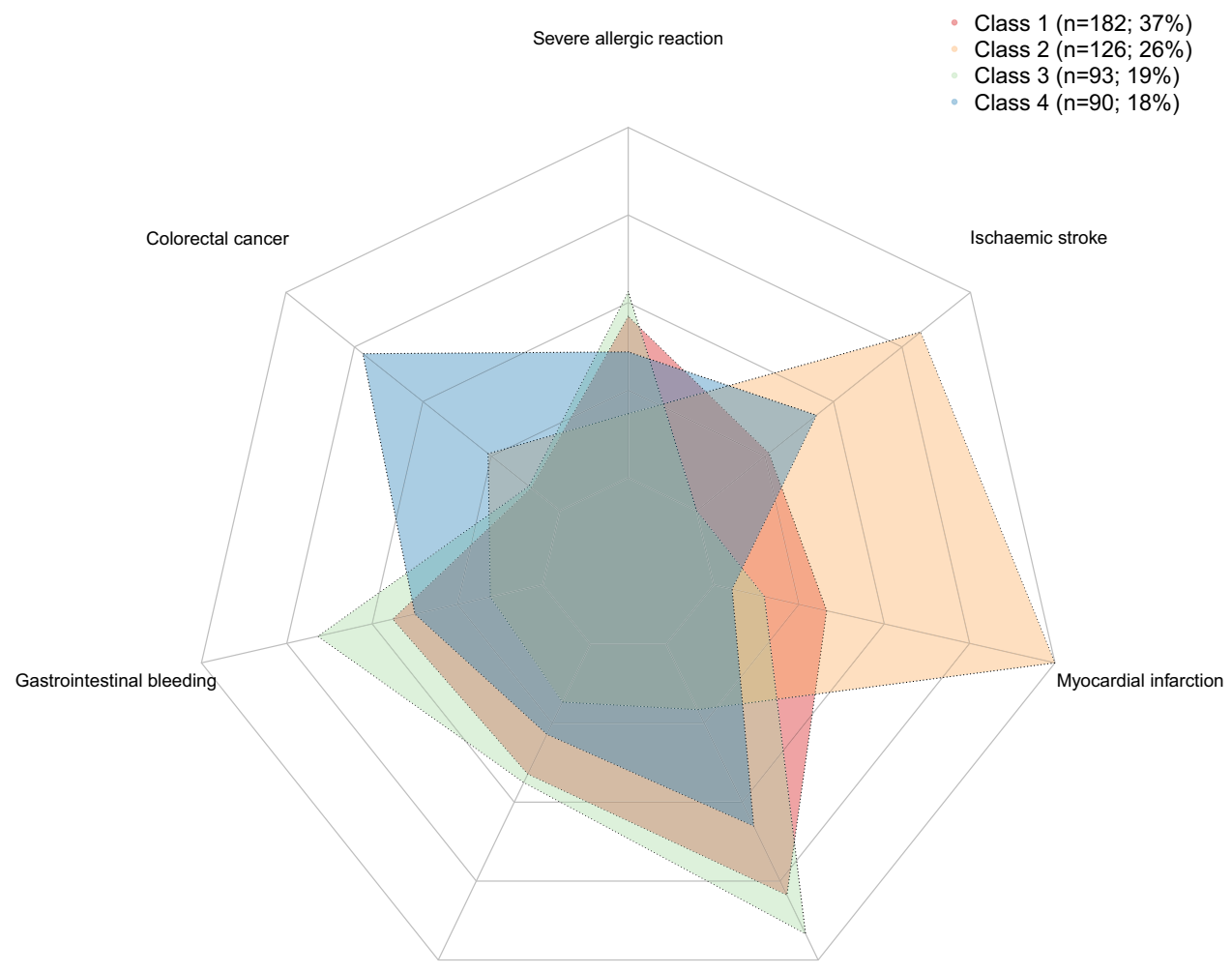

Peptic ulcer

Intracranial bleeding aspirin varied significantly both between and within the primary and secondary CVD prevention study groups. Nevertheless, seven discrete classes of preferences could be distinguished. Preventing myocardial infarction and ischaemic stroke were the most important treatment attributes for a class of participants in the primary CVD prevention group whose rate of current low-dose aspirin use was higher compared to other classes, possibly because they were aware of being at particularly high risk for these events. We also found that avoiding intracranial and gastrointestinal bleeding were most important treatment attributes to a class of participants in the secondary CVD prevention group with higher rates of several comorbidities and of hospitalization, indicating that they were less concerned about benefits than risks even though many of them had already experienced angina or a CV event. This could be due to the participants being concerned about the burden of further hospitalizations or additional comorbidities. Avoiding an increased risk of intracranial bleeding was the most important treatment attribute to another class of participants in the secondary CVD prevention group.

An added probable benefit of low-dose aspirin, not included in previous DCE low-dose aspirin studies, is protection against CRC [3-5]. A previous non-DCE study found that the CRC prevention benefit increased the acceptability of aspirin [40]. We found that the additional CRC prevention benefit was more important for patients in the primary CVD prevention group than in the secondary CVD prevention group. Moreover, one class of participants in the primary CVD prevention group, who tended to be younger, considered CRC prevention the most important treatment attribute.

The LCL models used in this study provide important information about the preferences of individuals taking lowdose aspirin for primary or secondary prevention of CVD; however, LCL models have some limitations [41]. First, as with all limited dependent variable models, the preference estimates are confounded with the utility scale, such that two classes may represent the same preferences but with different scales. However, we did not detect major scaling issues and we computed RI, which is a scale-free measure of preferences. Second, while the information obtained with LCL models provides insight into the preferences of different groups of patients at the population level, it cannot be used to guide treatment selection for individual patients. Another limitation of this study is that it relied on self-reporting of medical history and current and past treatments by participants. Any inaccuracies in the information provided by participants may have resulted in errors in assigning them to the primary and secondary CVD prevention groups and in the analyses of treatment preferences according to clinical characteristics. However, such errors would be unlikely to affect the finding that all latent classes considered the 
Fig. 4. Relative importance by preference class for the secondary cardiovascular disease prevention group

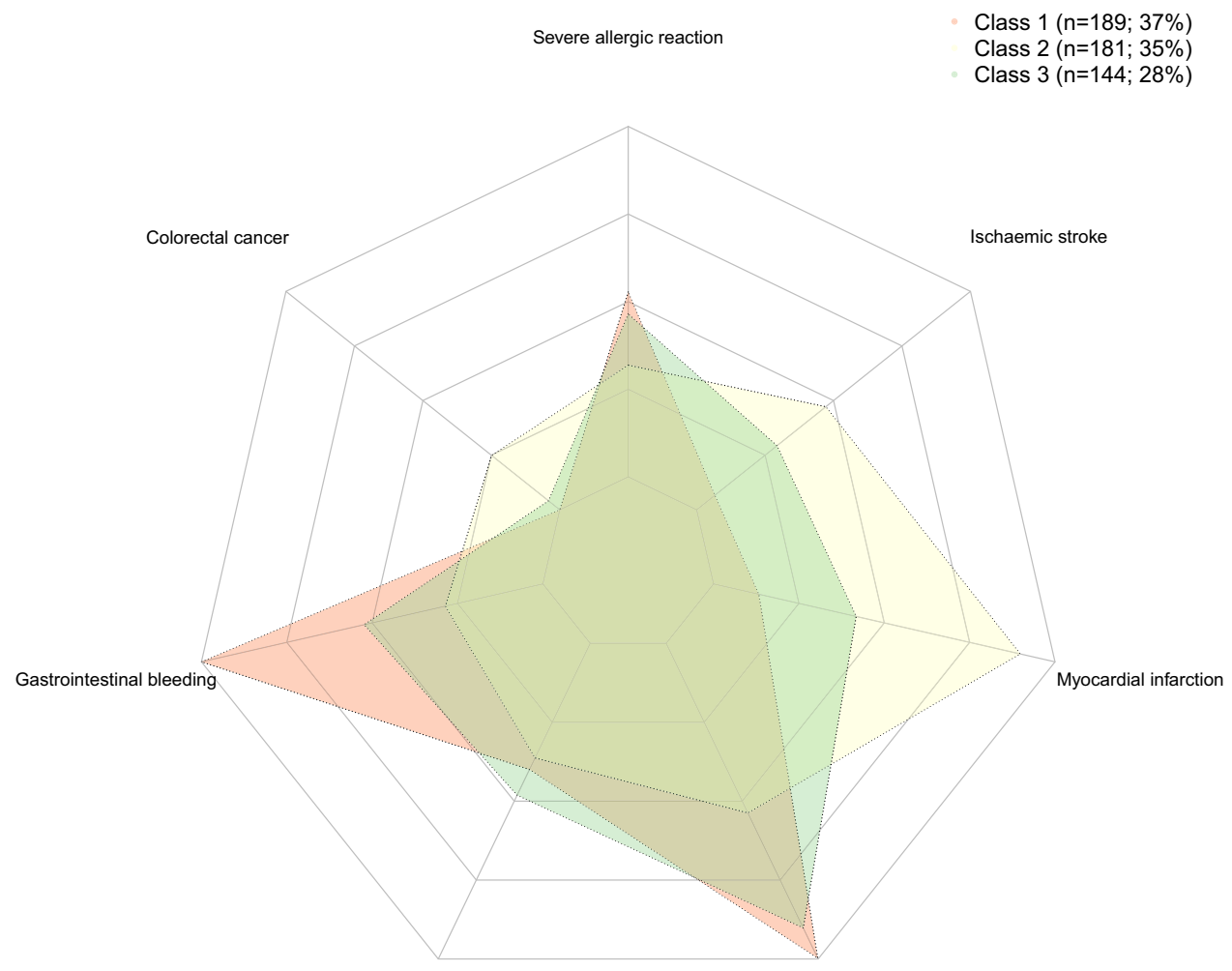

Peptic ulcer

Intracranial bleeding potential for adverse effects important. A further potential limitation is that differences in the strength of evidence for the potential benefits and risks of low-dose aspirin treatment (with evidence for prevention of myocardial infarction being stronger than that for CRC prevention, for example) were not explained to participants. Also, the adverse effect attribute levels were simply "increased risk" and "no risk", with no explicit consideration of changes across the probability scale. Moreover, some participants may not have given the survey questions adequate consideration. We reduced the potential impact of this by including a number of irrelevant options for some screening questions and screening out people who did not carefully consider all the listed options, for example, by indicating that they were taking an antidepressant for their heart diseases. Also, 94\% of participants answered the dominance question correctly, a rate similar to other DCEs in the health domain [42]. This suggests that the participants paid sufficient attention to the survey. The overall participation rate of 20\% (1005/5134) may have induced selection bias, but it is higher than for other patient preference studies with panel recruitment $[18,43]$. Furthermore, only a third of the study participants had adequate health literacy, which may have limited their understanding of the study materials. However, similarly low levels of health literacy were observed in another study in Italy [44], and no major issues with the final survey were detected in cognitive pilot interviews. Finally, this study was performed in a single country with only a quarter of the participants having a college education. Moreover, the primary CVD prevention study group was considerably younger than the general Italian population using low-dose aspirin for primary prevention of CVD [45], partially because people older than 75 years were excluded. Our results may therefore not be generalizable to other countries or populations.

\section{Conclusion}

Adherence to low-dose aspirin regimes is suboptimal, and patient education is imperative for achieving improvements in adherence [46]. Understanding how treatment preferences vary between individuals can help health care professionals to better communicate information on those benefits and risks of low-risk aspirin that matter most to a particular patient. This study indicated that the potential CRC prevention benefit may be especially important to some younger patients eligible for low-dose aspirin for primary prevention of CVD, and that many patients eligible for low-dose aspirin for primary or secondary CVD prevention are likely to be concerned by potential adverse effects of treatment. Translation of these insights to decision making in the clinic has the 
potential to improve patient education, which may ultimately lead to better treatment outcomes.

Supplementary Information The online version contains supplementary material available at https://doi.org/10.1007/s40271-021-00506-2.

Acknowledgements We thank Stephen Gilliver (Evidera) and Phil Leventhal (Evidera) for manuscript writing support, which was paid for by Bayer AG; Yingkai Cheng (formerly Bayer), Leo Plouffe (Bayer), Aasia Bhatti (Bayer), Elmar Detering (Bayer), Juliana Hirayama (Bayer), Akos Ferenc Pap (Bayer), Renata Martins (Bayer), Siir Su Saydam (Bayer), and Thomas Volkmer (Bayer) for informing study design and for consultations about the safety profile of low-dose aspirin; and Professor John Bridges (Ohio State University) for advice on study design.

\section{Declarations}

Funding This work was funded by Bayer AG.

Conflict of interest Pareen Vora, Montse Soriano Gabarró, and U1rike Wissinger are employees of Bayer. Tommi Tervonen, Jaein Seo, Nicolas Krucien, and Kevin Marsh are employees of Evidera, which received funding from Bayer to conduct the research. Raffaele De Caterina has received fees from Boehringer Ingelheim, Bayer, BristolMyers Squibb/Pfizer, Daiichi Sankyo, AstraZeneca, Sanofi-Aventis, Eli Lilly, and Novartis; and grant support from Boehringer Ingelheim, Bayer, Bristol-Myers Squibb/Pfizer, Daiichi Sankyo, and AstraZeneca.

Ethics approval Ethical approval for the study was granted by Ethical \& Independent Review Services institutional review board (Independence, MO, USA) before any study procedures were initiated (institutional review board reference number 18118-01 for initial submission, 18118-02 for continuing review, and 18118-02A for additional recruitment templates for main data collection).

Consent All individual participants included in the study provided online consent to participate.

Availability of data and material The datasets generated during and/ or analysed during the current study are not publicly available as no consent was sought from participants to allow sharing of data with third parties.

\section{Code availability Not applicable.}

Author contributions TT contributed to the conception, design, and implementation of the work and analysis, interpretation of data for the work, and drafting and revision of the manuscript. PV contributed to the conception, design, and implementation of the work, interpretation of data for the work, and revision of the manuscript. JS contributed to the design and implementation of the work, acquisition and interpretation of data for the work, and drafting and revision of the manuscript. NK contributed to analysis, interpretation of data for the work, and revision of the manuscript. KM contributed to the design and implementation of the work, and revision of the manuscript. RDC contributed to interpretation of data for the work and revision of the manuscript. UW contributed to design of the work, and revision of the manuscript. MSG contributed to the conception, design and implementation of the work, interpretation of data for the work, and revision of the manuscript. All gave final approval and agree to be accountable for all aspects of work ensuring integrity and accuracy.
Open Access This article is licensed under a Creative Commons Attribution-NonCommercial 4.0 International License, which permits any non-commercial use, sharing, adaptation, distribution and reproduction in any medium or format, as long as you give appropriate credit to the original author(s) and the source, provide a link to the Creative Commons licence, and indicate if changes were made. The images or other third party material in this article are included in the article's Creative Commons licence, unless indicated otherwise in a credit line to the material. If material is not included in the article's Creative Commons licence and your intended use is not permitted by statutory regulation or exceeds the permitted use, you will need to obtain permission directly from the copyright holder. To view a copy of this licence, visit http://creativecommons.org/licenses/by-nc/4.0/.

\section{References}

1. De Caterina R, Aimo A, Ridker PM. Aspirin therapy for primary prevention: the case for continuing prescribing to patients at high cardiovascular risk-a review. Thromb Haemost. 2020;120(2):199-206.

2. Cosentino F, Grant PJ, Aboyans V, Bailey CJ, Ceriello A, Delgado V, et al. 2019 ESC Guidelines on diabetes, pre-diabetes, and cardiovascular diseases developed in collaboration with the EASD. Eur Heart J. 2020;41(2):255-323.

3. Cook NR, Lee IM, Zhang SM, Moorthy MV, Buring JE. Alternate-day, low-dose aspirin and cancer risk: long-term observational follow-up of a randomized trial. Ann Intern Med. 2013;159(2):77-85.

4. Friis S, Riis AH, Erichsen R, Baron JA, Sorensen HT. Lowdose aspirin or nonsteroidal anti-inflammatory drug use and colorectal cancer risk: a population-based case-control study. Ann Intern Med. 2015;163(5):347-55.

5. Rothwell PM, Wilson M, Elwin CE, Norrving B, Algra A, Warlow $\mathrm{CP}$, et al. Long-term effect of aspirin on colorectal cancer incidence and mortality: 20-year follow-up of five randomised trials. Lancet. 2010;376(9754):1741-50.

6. Cuzick J, Thorat MA, Bosetti C, Brown PH, Burn J, Cook NR, et al. Estimates of benefits and harms of prophylactic use of aspirin in the general population. Ann Oncol. 2015;26(1):47-57.

7. Bibbins-Domingo K. Aspirin use for the primary prevention of cardiovascular disease and colorectal cancer: U.S. preventive services task force recommendation statement. Ann Intern Med 2016;164(12):836-45.

8. Berkes EA. Anaphylactic and anaphylactoid reactions to aspirin and other NSAIDs. Clin Rev Allergy Immunol. 2003;24(2):137-48.

9. Whitlock EP, Williams SB, Burda BU, Feightner A, Beil T. Aspirin use in adults: cancer, all-cause mortality, and harms: a systematic evidence review for the US Preventive Services Task Force. Rockville (MD); 2015.

10. Whitlock EP, Burda BU, Williams SB, Guirguis-Blake JM, Evans CV. Bleeding risks with aspirin use for primary prevention in adults: a systematic review for the U.S. Preventive Services Task Force. Ann Intern Med. 2016;164(12):826-35.

11. Halvorsen S, Andreotti F, ten Berg JM, Cattaneo M, Coccheri S, Marchioli R, et al. Aspirin therapy in primary cardiovascular disease prevention: a position paper of the European Society of Cardiology working group on thrombosis. J Am Coll Cardiol. 2014;64(3):319-27.

12. Puhan MA, Yu T, Stegeman I, Varadhan R, Singh S, Boyd CM. Benefit-harm analysis and charts for individualized and preference-sensitive prevention: example of low dose aspirin for primary prevention of cardiovascular disease and cancer. BMC Med. 2015;1(13):250. 
13. Dehmer SP, Maciosek MV, Flottemesch TJ, LaFrance AB, Whitlock EP. Aspirin for the primary prevention of cardiovascular disease and colorectal cancer: a decision analysis for the U.S. Preventive Services Task Force. Ann Intern Med. 2016;164(12):777-86.

14. Johnson FR, Zhou M. Patient preferences in regulatory benefit-risk assessments: a U.S. perspective. Value Health. 2016;19(6):741-5.

15. Juhaeri J. Benefit-risk evaluation: the past, present and future. Ther Adv Drug Saf. 2019;10:2042098619871180.

16. Tervonen T, Angelis A, Hockley K, Pignatti F, Phillips LD. Quantifying preferences in drug benefit-risk decisions. Clin Pharmacol Ther. 2019;106(5):955-9.

17. Ghijben P, Lancsar E, Zavarsek S. Preferences for oral anticoagulants in atrial fibrillation: a best-best discrete choice experiment. Pharmacoeconomics. 2014;32(11):1115-27.

18. Najafzadeh M, Gagne JJ, Choudhry NK, Polinski JM, Avorn J, Schneeweiss SS. Patients' preferences in anticoagulant therapy: discrete choice experiment. Circ Cardiovasc Qual Outcomes. 2014;7(6):912-9.

19. Muhlbacher AC, Bethge $\mathrm{S}$. Reduce mortality risk above all else: a discrete-choice experiment in acute coronary syndrome patients. Pharmacoeconomics. 2015;33(1):71-81.

20. Stafinski T, Menon D, Nardelli A, Bakal J, Ezekowitz J, Tymchak W, et al. Incorporating patient preferences into clinical trial design: results of the opinions of patients on treatment implications of new studies (OPTIONS) project. Am Heart J. 2015;169(1):122-31 e22.

21. Najafzadeh M, Schneeweiss S, Choudhry NK, Avorn J, Gagne JJ. General population vs. patient preferences in anticoagulant therapy: a discrete choice experiment. Patient. 2019;12(2):235-46.

22. Ryan M, Farrar S. Using conjoint analysis to elicit preferences for health care. BMJ. 2000;320(7248):1530-3.

23. Soekhai V, de Bekker-Grob EW, Ellis AR, Vass CM. Discrete choice experiments in health economics: past, present and future. Pharmacoeconomics. 2019;37(2):201-26.

24. Bridges JF, Hauber AB, Marshall D, Lloyd A, Prosser LA, Regier DA, et al. Conjoint analysis applications in health-a checklist: a report of the ISPOR Good Research Practices for Conjoint Analysis Task Force. Value Health. 2011;14(4):403-13.

25. Coast J, Al-Janabi H, Sutton EJ, Horrocks SA, Vosper AJ, Swancutt DR, et al. Using qualitative methods for attribute development for discrete choice experiments: issues and recommendations. Health Econ. 2012;21(6):730-41.

26. Cardiovascular risk chart. 2008 [cited 202029 January]. http:// www.cuore.iss.it/eng/assessment/chart.asp.

27. Chew LD, Bradley KA, Boyko EJ. Brief questions to identify patients with inadequate health literacy. Fam Med. 2004;36(8):588-94.

28. Jornay PM. Evening-dosed methylphenidate for ADHD. Med Lett Drugs Ther. 2019;61(1578):126-8.

29. R: a language and environment for statistical computing. 2013 [cited 202017 December]. http://www.R-project.org/.

30. Croissant Y. Estimation of random utility models in R: the mlogit package. J Stat Softw. 2020;95(11):1-41.
31. Sarrias M, Daziano R. Multinomial logit models with continuous and discrete individual heterogeneity in R: the gmnl package. J Stat Softw. 2017;79(2):1-46.

32. Krinsky I, Robb AL. On approximating the statistical properties of elasticities. Rev Econ Stat. 1986;68:715-9.

33. Krinsky I, Robb AL. On approximating the statistical properties of elasticities: a correction. Rev Econ Stat. 1990;72:189-90.

34. Devereaux PJ, Anderson DR, Gardner MJ, Putnam W, Flowerdew GJ, Brownell BF, et al. Differences between perspectives of physicians and patients on anticoagulation in patients with atrial fibrillation: observational study. BMJ. 2001;323(7323):1218-22.

35. Yuan Z, Levitan B, Burton P, Poulos C, Brett Hauber A, Berlin JA. Relative importance of benefits and risks associated with antithrombotic therapies for acute coronary syndrome: patient and physician perspectives. Curr Med Res Opin. 2014;30(9):1733-41.

36. Myles PS, Thompson G, Fedorow C, Farrington C, Sheridan N. Evaluation of differences in patient and physician perception of benefit and risks of aspirin and antifibrinolytic therapy in cardiac surgery. Anaesth Intensive Care. 2014;42(5):592-8.

37. Okumura K, Inoue H, Yasaka M, Gonzalez JM, Hauber AB, Levi$\tan \mathrm{B}$, et al. Comparing patient and physician risk tolerance for bleeding events associated with anticoagulants in atrial fibrillation-evidence from the United States and Japan. Value Health Reg Issues. 2015;6:65-72.

38. Shafrin J, Bruno A, MacEwan JP, Campinha-Bacote A, Trocio J, Shah M, et al. Physician and patient preferences for nonvalvular atrial fibrillation therapies. Value Health. 2016;19(4):451-9.

39. Laba TL, Howard K, Rose J, Peiris D, Redfern J, Usherwood $\mathrm{T}$, et al. Patient preferences for a polypill for the prevention of cardiovascular diseases. Ann Pharmacother. 2015;49(5):528-39.

40. Hur C, Broughton DE, Kong CY, Ozanne EM, Richards EB, Truong T, et al. Patient preferences for the chemoprevention of colorectal cancer. Dig Dis Sci. 2009;54(10):2207-14.

41. Hauber AB, Gonzalez JM, Groothuis-Oudshoorn CG, Prior T, Marshall DA, Cunningham C, et al. Statistical methods for the analysis of discrete choice experiments: a report of the ISPOR Conjoint Analysis Good Research Practices Task Force. Value Health. 2016;19(4):300-15.

42. Tervonen T, Schmidt-Ott T, Marsh K, Bridges JFP, Quaife M, Janssen E. Assessing rationality in discrete choice experiments in health: an investigation into the use of dominance tests. Value Health. 2018;21(10):1192-7.

43. Tervonen T, Hawken N, Hanania NA, Martinez FJ, Heidenreich $S$, Gilbert I. Maintenance inhaler therapy preferences of patients with asthma or chronic obstructive pulmonary disease: a discrete choice experiment. Thorax. 2020;75(9):735-43.

44. Schiavone S, Attena F. Measuring Health Literacy in Southern Italy: a cross-sectional study. PLoS ONE. 2020;15(8):e0236963.

45. Manes C, Giacci L, Sciartilli A, D’Alleva A, De Caterina R. Aspirin overprescription in primary cardiovascular prevention. Thromb Res. 2006;118(4):471-7.

46. Duffy D, Kelly E, Trang A, Whellan D, Mills G. Aspirin for cardioprotection and strategies to improve patient adherence. Postgrad Med. 2014;126(1):18-28. 